\title{
ACCESSING THE ACCELERATION OF THE UNIVERSE WITH SUNYAEV-ZELDOVICH AND X-RAY DATA FROM GALAXY CLUSTERS
}

\author{
RODRIGO F. L. HOLANDA, JOSÉ A. S. LIMA*, JOÃO V. CUNHA \\ Departamento de Astronomia, Universidade de São Paulo \\ Rua do Matão, 1226 - 05508-900, São Paulo, SP, Brazil, \\ *E-mail:limajas@astro.iag.usp.br
}

\begin{abstract}
By using exclusively the Sunyaev-Zel'dovich effect and X-ray surface brightness data from 25 galaxy clusters in the redshift range $0.023 \leq z \leq 0.784$ we access cosmic acceleration employing a kinematic description. Such result is fully independent on the validity of any metric gravity theory, the possible matter-energy contents filling the Universe, as well as on the SNe Ia Hubble diagram.
\end{abstract}

Keywords: Sunyaev-Zeldovich Effect, Galaxy Clusters, Accelerated Universe.

\section{Introduction}

Currently, SNe type Ia provides a unique direct access to the late time accelerating stage of the Universe. ${ }^{1}$ Naturally, this a rather uncomfortable situation from the observational and theoretical viewpoints. A promising distance estimator fully independent of SNe type Ia and other calibrators of the cosmic distance ladder is the angular diameter distance $\left(D_{A}(z)\right)$ from a given set of distant objects. ${ }^{2}$ It has also been recognized that the combination of $\mathrm{SZE}^{3}$ and X-ray surface brightness measurements may provide useful angular diameters from galaxy clusters. ${ }^{4-6}$

De Filippis et al. $(2005)^{6}$ reanalyzed and derived, using an isothermal elliptical 2 -Dimensional $\beta$-model to describe the clusters, $\mathcal{D}_{A}$ measurements for 25 clusters from two previous compilations, ${ }^{7,8}$ where was used a spherical isothermal $\beta$ model to describe the clusters geometry. More recently, it was shown ${ }^{9,10}$ that De Filippis et al. sample is in good agreement with the distance duality (DD) relation between luminosity distance $\left(\mathcal{D}_{L}\right)$ and angular diameter distance $\left(\mathcal{D}_{A}\right), \mathcal{D}_{L}(1+z)^{-2} / \mathcal{D}_{A}=1$, in the context of a $\Lambda$ CDM model (WMAP7). ${ }^{11}$ This sample is also consistent ( $2 \sigma$ c.l.) with no violation of the DD relation in a model-independent cosmological test involving $D_{A}$ from galaxy clusters and $D_{L}$ from the supernovae Ia data provided by the Constitution compilation. ${ }^{12}$

In this work, we investigate the potentialities of SZE/X-ray technique by employing a purely kinematic description of the universal expansion based on angular diameter distances of clusters for two different expansions of the deceleration pa- 
rameter $^{13}: q(z)=q_{0}+q_{1} z$ and $q(z)=q_{0}+q_{1} z /(1+z)$. As we shall see, by using the De Filippis et al. ${ }^{6}$ sample we find that a kinematic analysis based uniquely on cluster data suggests that the Universe is accelerating today, such as, $q_{0}<0$ with $83 \%$ of probability.

\section{Kinematic Approach and Constraints}

Let us now assume that the Universe is spatially flat as motivated by inflation and WMAP measurements. ${ }^{11}$ In this case, the angular diameter distance in the FRW metric is defined by (in our units $c=1$ ),

$$
\begin{aligned}
D_{A}= & (1+z)^{-1} H_{0}^{-1} \int_{0}^{z} \frac{d u}{H(u)}=\frac{(1+z)^{-1}}{H_{0}} \\
& \int_{0}^{z} \exp \left[-\int_{0}^{u}[1+q(u)] d \ln (1+u)\right] d u,
\end{aligned}
$$

where $H(z)=\dot{a} / a$ is the Hubble parameter, and, $q(z)$, the deceleration parameter, is defined by

$$
q(z) \equiv-\frac{a \ddot{a}}{\dot{a}^{2}}=\frac{d H^{-1}(z)}{d t}-1 .
$$

Let us now consider the 25 measurements of angular diameter distances from galaxy clusters as obtained through SZE/X-ray method by De Filippis and coworkers. ${ }^{6}$ In our analysis we use a maximum likelihood determined by a $\chi^{2}$ statistics

$$
\chi^{2}(z \mid \mathbf{p})=\sum_{i} \frac{\left(\mathcal{D}_{A}\left(z_{i} ; \mathbf{p}\right)-\mathcal{D}_{A o, i}\right)^{2}}{\sigma_{\mathcal{D}_{A o, i}}^{2}+\sigma_{s y s .}^{2}},
$$

where $\mathcal{D}_{A o, i}$ is the observational angular diameter distance, $\sigma_{\mathcal{D}_{A o, i}}$ is the uncertainty in the individual distance, $\sigma_{s y s}$ is the contribution of the systematic errors and the complete set of parameters is given by $\mathbf{p} \equiv\left(H_{0}, q_{0}, q_{1}\right)$. We have marginalized on the Hubble Distance $\left(H_{0}^{-1}\right)$ with a gaussian prior on $H_{0}$ centered in our best fit value $H_{0}=77 \pm 4 \mathrm{~km} / \mathrm{s} / \mathrm{Mpc}$.

\section{Results and Discussion}

In Fig. 1(a) we show the contour in the plane $q_{0}-q_{1}$ for linear parametrization. The confidence region $(1 \sigma)$ are $-3.2 \leq q_{0} \leq 0.7$ and $29 \leq q_{1} \leq-9$. In Fig. 1(b) we show the likelihood for $q_{0}$ (solid line-only statistical errors and dashed linestatistical + systematic errors). We have marginalized on the Hubble distance with a gaussian prior on $H_{0}$ centered in its best fit $H_{0}=77 \pm 4 \mathrm{~km} / \mathrm{s} / \mathrm{Mpc}$ and on all values of $q_{1}$. We obtain that $q_{0}<0$ with $84 \%$ of probability, $q_{0}=-1.29_{-1.5}^{+1.31}$ $\left(1 \sigma\right.$ - only statistical errors) and $74 \%$ of probability, $q_{0}=-0.8_{-1.7}^{+1.6}(1 \sigma$ - statistical + systematic errors). In Figs. 1(c) and 1(d) we display the corresponding plots for the non linear parameterization. The confidence region $(1 \sigma)$ is now defined by: 
$-3.5 \leq q_{0} \leq 0.9$ and $-17 \leq q_{1} \leq 27$. In Fig. 1 (d) we show the likelihood distribution function for $q_{0}$. We obtain that $q_{0}<0$ with $82 \%$ of probability, $q_{0}=-1.4_{-1.6}^{+1.41}(1 \sigma$ - only statistical errors) and with $72 \%$ of probability, $q_{0}=-1_{-1.6}^{+1.7}(1 \sigma$ - statistical + systematic errors).
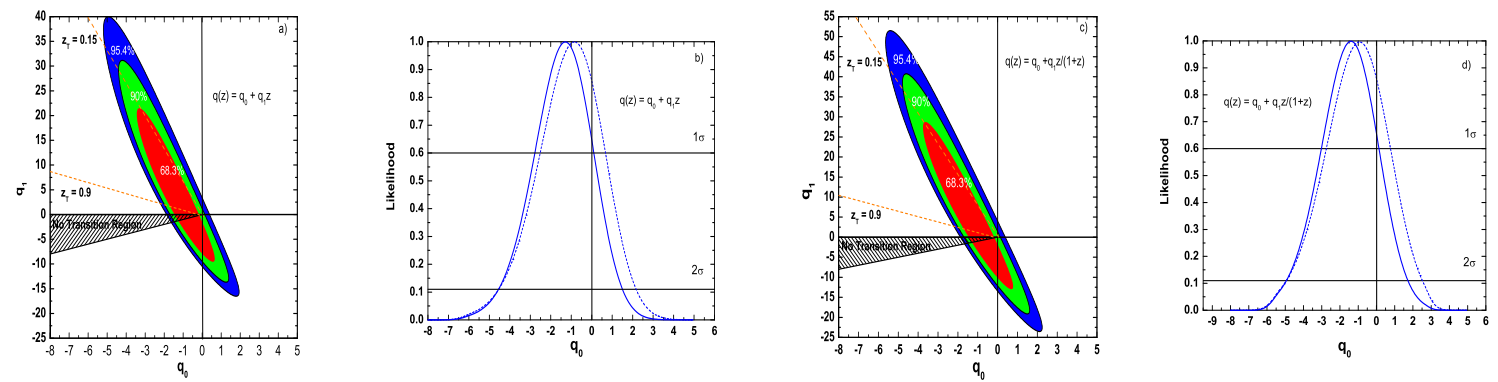

Fig. $1 . \quad$ a) Contours in the $q_{o}-q_{1}$ plane for 25 galaxy clusters data ${ }^{6}$ considering $q(z)=q_{0}+q_{1} z$. b) Likelihood function for the $q_{0}$, marginalizing on all values of $q_{1}$. c) Contours in the $q_{o}-q_{1}$ plane considering $q(z)=q_{0}+q_{1} z /(1+z)$. d) Likelihood function for the $q_{0}$, marginalizing on all values of $q_{1}$ for non-linear parametrization.

We have shown that the combination of Sunyaev-Zeldovich/X-ray data from galaxy clusters is an interesting technique for accessing the present accelerating stage of the Universe. This result follows from a consistent kinematic approach based on the angular diameter distance of galaxy clusters obtained from SZE/X-ray measurements. By using two different parameterizations, it was found that $q_{0}<0$ with at least $83 \%$ probability (only statistical errors in galaxy clusters data) and $72 \%$ probability (statistical + systematic errors in galaxy clusters data).

\section{References}

1. A. G. Riess et al., Astron. J. 116, 1009 (1998); S. Perlmutter et al., ApJ 517, 565 (1999).

2. L. I. Gurvits et al., A\&A 342, 378 (1999); J. A. S. Lima \& J. S. Alcaniz, A\&A 357, 393 (2000), astro-ph/0003189; ibdem, ApJ 566, 15 (2002), astro-ph/0109047.

3. R. A. Sunyaev \& Ya. B. Zel'dovich, Astrophys. Space Sci. 7, 20 (1970); ibdem, Comments Astrophys. Space Phys. 4, 173 (1972).

4. A. Cavaliere, R. Fusco-Fermiano, A\&A 70, 677 (1978).

5. J. V. Cunha, L. Marassi \& J. A. S. Lima, MNRAS 379, L1 (2007), astro-ph/0611934.

6. E. De Filippis, M. Sereno, M. W. Bautz \& G. Longo, ApJ 625, 108 (2005).

7. B. S. Mason et al., ApJ 555, L11 (2001).

8. E. D. Reese et al., ApJ 581, 53 (2002).

9. R. F. L. Holanda, J. A . S. Lima \& M. B. Ribeiro, A\&A, 528, L14 (2011).

10. R. F. L. Holanda, J. A . S. Lima \& M. B. Ribeiro, ApJL, 722, L233 (2010).

11. Komatsu, E. et al. Astrophys. J. Suplem., 192, 18 (2011).

12. M. Hicken et al., ApJ, 700, 1097 (2009).

13. J. V. Cunha \& J. A. S. Lima, MNRAS 390, 210 (2008), arXiv:0805.1261. 